- No. $68-284$
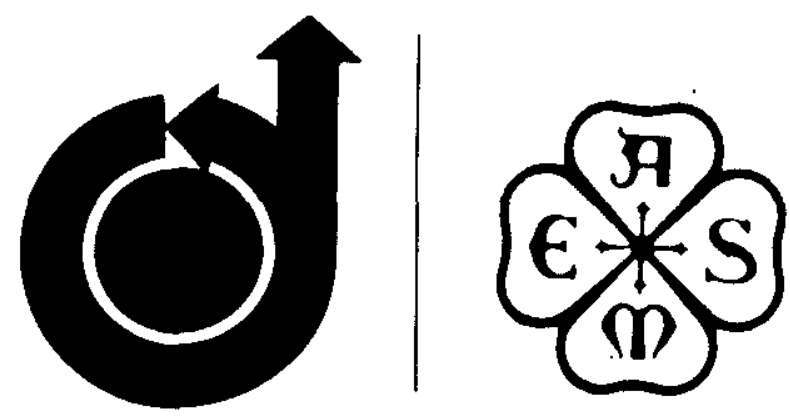

\title{
ENGINEERING ESTIMATES FOR SUPERSONIC FLUTTER OF CURVED SHELL SEGMENTS
}

by

W. J. ANDERSON and K-H. HSU

The University of Michigan

Ann Arbor, Michigan

AlAA Paper

No. 68-284

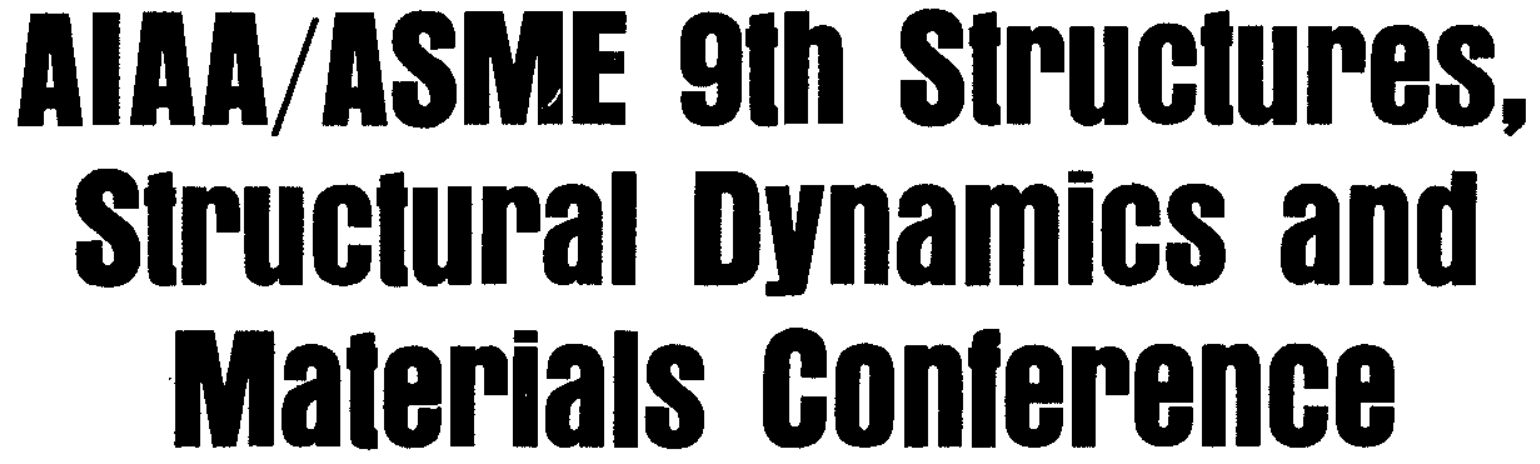

PALM SPRINGS, CALIFORNIA/APRIL 1.3, 1968 


\title{
ENGINEERING ESTIMATES FOR SUPERSONIC FLUTTER
}

OF CURVED SHELL SEGMENTST

\author{
William J. Anderson* and Kuo-Hsiung Hsu** \\ The University of Michigan \\ Ann Arbor, Michigan
}

\section{Abstract}

Theoretical flutter boundaries are given for cylindrical shell segments. The problem was motivated by portions of the Saturn $V$ booster. Donnell's cylinder equations are used in conjunction with Galerkin's method. The aerodynamic expression is simplified by using a static theory. This theory, however, includes a parameter which typifies the spatial pressure distribution. As the parameter is varied, the pressure distribution changes from that given by Ackeret theory to that typical of a steady, slender body theory. These extremes are felt to provide physical bounds for the types of flow over an isolated shell segment. The result is an upper and lower estimate for the flutter boundary, yielding a thickness requirement as a function of panel curvature and length-towidth ratio. The segments are less sensitive to the spatial pressure distribution than expected. I'his may account for the relative success of Ackeret theory in predicting cylinder flutter to date.

\section{Nomenclature}

\begin{tabular}{|c|c|}
\hline$D$ & $\operatorname{En} 3 /\left[12\left(1-v^{2}\right)\right]$ \\
\hline$F$ & Alry stress function \\
\hline $\mathrm{H}$ & $\begin{array}{l}\text { Thickness parameter, } \\
{\left[[M-1]^{1 / 2}{ }^{2} /\left[\left(1-v^{2}\right) q\right]\right]^{1 / 3} \mathrm{~h} / \mathrm{L}}\end{array}$ \\
\hline b & Panel Thlckness \\
\hline Y & Length of panel \\
\hline$y$ & Mach number \\
\hline$x$ & Axial wavé number \\
\hline iv & Number of modes \\
\hline $\mathrm{w}_{x}, \mathrm{~N}_{\mathrm{z}}$ & $\begin{array}{l}\text { Stress resultants, see equations ( } 5) \\
\text { and }(6)\end{array}$ \\
\hline$r(x, \theta, t)$ & Aerodynamic load \\
\hline a & Integer, also dynamic pressure \\
\hline U! & Radius \\
\hline$t$ & Time \\
\hline$y$ & Flow velocity \\
\hline ॠ & Width of panel \\
\hline$\dot{x}_{\in f f}$ & Effect1ve width of panel, $\mathrm{W} / \mathrm{n}$ \\
\hline & Panel displacement in radial direction \\
\hline $\mathrm{x}$ & Spatial coordinate, flow direction \\
\hline R & Curvature parameter, $(L / R)(L / h) \sqrt{1-v^{2}}$ \\
\hline$\delta \mathrm{ym}$ & Kronecker Delta \\
\hline 24 & Angular coordinate \\
\hline$\theta_{0}$ & Included angle of shell. segment \\
\hline$\pi$ & Eigenvalue \\
\hline 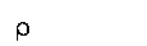 & Fluid density \\
\hline
\end{tabular}

$\rho_{s}$ Panel density

$\psi$ Spatial phase shift

w Frequency, rad/sec

\section{Introduction}

This theoretical study concerns the aeroelastic instability of a cylindrical shell segment. The problem was motivated by the need for design criteria for portions of the external structure of the Saturn $V$ booster. The panel is a rectangular plate bent to a cylindrical shape and is freely supported on all four sides (Figure 2 ). It is surrouncied by sigid structure (Figure 2). Super-

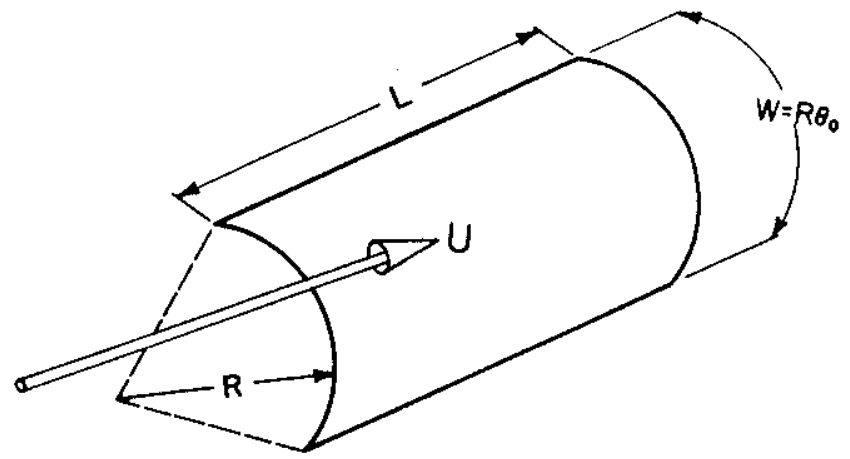

Figure 1. Shell segment.

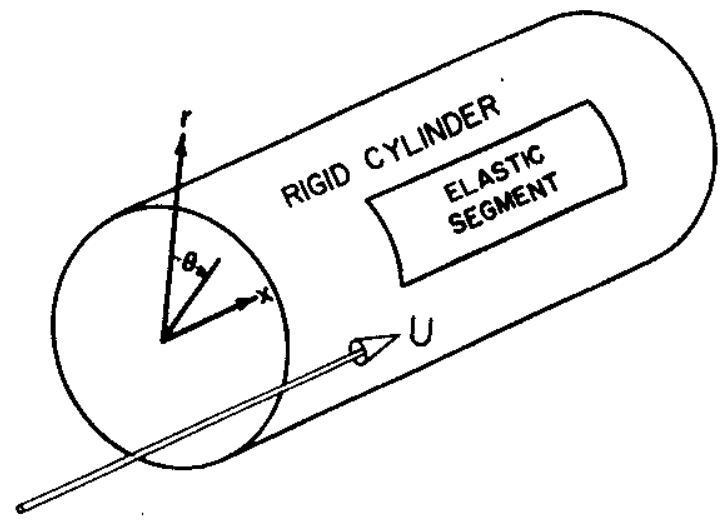

Figure 2. Elastic shell segment imbedded in a cylinder.

TThis work was supported under NASA Grant NGR-23-005-166

*Assistant Professor, Department of Aerospace Engineering

**Graduate Student, Department of Engineering Mechanics 
sonic flow is directed parallel to the generators of the cylindrical shell segment. primery interest is in low aspect ratio panels.

There is serious need for theoretical criteria for panel flutter and divergence of such shell segments. In the absence of such data, designers must either ignore the possibility of panel flutter on the structure, or accept its occurrence stoically with the hope that the missile will survive. .

The stmuctural side of the problem has been studied in a conventional way, with the use of Donneli's cylinder equations. Galerkin's method is used to find linear stability boundaries.

First attempts at the problem involved the use of potential flow aerodymanc theory. For such a panel, these relations are so difficult to handle that the calculation of "coupled mode" flutter boundaries is not practical at the present time. Furthernore, the more exact aerodynamic theories (which account for finite width and length as well as frequency dependence) would yield results which are not useful to the designer. Too many parameters enter.

On the other hand, Ackeret (linearized twoatmensional supersonic) theory has proven useful in predicting the only cylinder flutter experi. ments to date (Stearman, Lock, and Fung, and 0lson and Fung).(1,2) This is of some interest because for the wavelengths involved in the wind tunnel. tests, it is known that Ackeret theory results in pressure expressions which are in serious error concerning the spatial distribution of pressure.

In the present study, therefore, a static aerodymamic theory is used which is based on Ackeret theory. The aerodynamic pressures are taken to be of magnitude equal to that given by Ackeret theory but with an arbitrary parameter allowing a varying spatial pressure distribution.

In this manner, as the parameter is varied, the pressure expression changes continuously from a pressure distribution typical of Ackeret theory to one typical of a steady slender body aerodymamic theory. Potential theory suggests that for segments of an oscillating cylinder, the true spatial distribution should lie somewhere between these two extremes.

The inclusion of such a "correction" to Ackeret theory to allow for uncertainty in the aerodynamic pressure would be useless if the dynamic stability of the panel were sensitive to such a cholce. The results show, however, that the variation of the spatial pressure distribution has only a moderate effect on the stablity of the panel. Upper and lower estimates of the stability boundary can hence be given. The panel thickness requirements differ by less than 35\% over a wide range of parameters.
One advantage of this approach is that the results can be presented in an understandable (albeit somewhat oversimplified) manner. A thickness parameter required to prevent flutter is plotted versus a length-to-width ratio. A curvature parameter enters in much the same way as in cylinder buckling work. It is felt that the design parameters used here are somewhat universal and will prove to be useful in the long run, even after more precise theories are available. Corrections to the design curves can be made as more experimental data are obtained.

The results do not include unsteady aerodynamic effects and cannot predict the single degree-offreedom flutter typical of transonic flutter. Also, the approach used here is less accurate if used for pressurized shell segments, where frequenctes are higher.

Prevlous work has been done on related problems. Dzygadio studied the elastic instability of an infinitely long elastic segment of an infi-

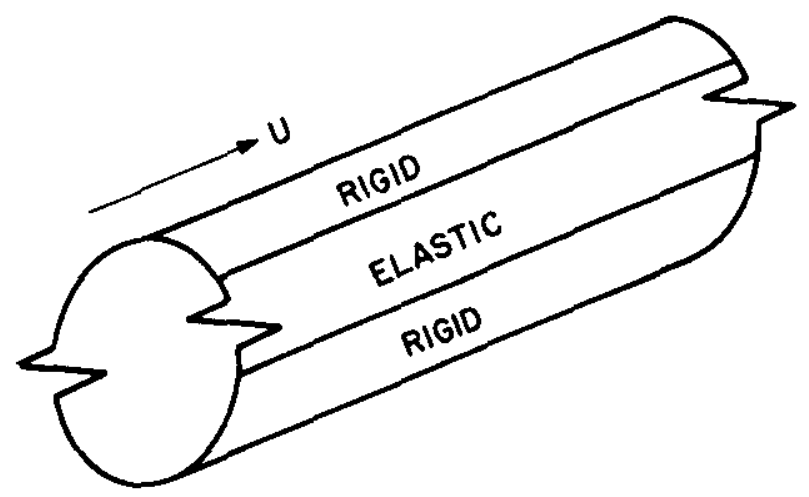

Figure 3. Cylinder studied by Dzygiadio.

nitely long cylinder. (3) The stability boundarles were found for a traveling wave:

$$
w(x, \theta, t)=w(\theta) e^{1 \alpha\left(x-v_{t}\right)}
$$

A set of integro-differential equations of motion resulted. These were solved with the aid of a Fourler series in the $\theta$ variable. Much effort was placed on a study of the effect of structural damping on the stability boundaries. For moderate amounts of damping, unexpected changes in the panel's stability resulted. The numerical results presented were not extensive. It was concluded that for small damping ratios and for fixed shell thickness and radius, the critical Mach number does not vary greatly for included angles for the segment lying between $\pi / 4$ and $\pi$. 
Another study of interest was by Dowell and Widnall. (4) The case considered was a finite length elastic segment in an infinitely long rigid cylindrical shell. In this case, the generalized aerodynamic forces were found for deflections of the type

$$
w(x, \theta, t)=e^{j \omega t} \cos n \theta \sin \frac{m \pi x}{\widetilde{I}} .
$$

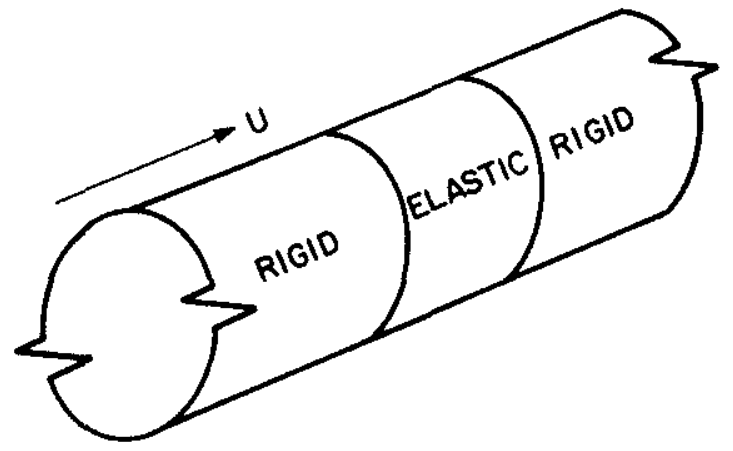

Figure 4. Cylinder studied by Dowell.

Dowell made several comments about the stability of the shell segment merely by looking at the character of the generalized forces. First of all, in the low supersonic Mach number range, a single degree-of-freedom type of flutter is possible. Secondly, for shell segments with long length-towidth ratios, static divergence takes place. Flutter boundarles for the "coupled-mode" type of flutter were not presented.

Another important study was done by Randall, who calculated pressure distributions on stationary, three-dimensional "bumps" (such as canopies) attached to cylinders. (5) The work dealt only with drag and the question of elastic stability of the canopy was not studied.

The present solution parallels the approach used by McElman to some extent. (6) McElman studied a curved orthotropic panel segment by using a two-mode analysis with Ackeret theory. No design curves of the type shown here were presented in McElman's work. Also, it is necessary to use many more than two modes when dealing with low aspect ratio panels.

\section{Ir. Problem Statement}

Consider a cylindrical shell segment as shown in Figure 1. Supersonic flow passes over the outer surface of the segment, with flow direction parallel to the cylinder axis. The segment is of uniform thickness and of isotropic, homogeous elastic material. Conventional cylindrical coordinates $x, r, \theta$ are used. The shell segment is defined by

$$
\begin{gathered}
r=R \\
0 \leq x \leq \dot{L} \\
\frac{-\theta_{0}}{2} \leq \theta \leq \frac{-\theta_{0}}{2}
\end{gathered}
$$

Deflection of the surface of the segment will be given by $w(x, \theta, t)$ measured from the mean radius of the shell. The edges of the shell will be "freelysupported" as defined below. The shell may be internally pressurized. No structural damping will be included.

\section{Structural Details}

The shell is thin and inftialiy circular. Radial deflections are restricted to be small:

$$
\frac{w(x, \theta, t)}{h} \ll 1
$$

The in-plane motions of the shell $u(x, \theta, t)$ and $v(x, \theta, t)$ are small compared to $w(x, \theta, t)$ so that Inertial effects due to in-plane motion can be neglected (Relssner's assumption). The included angle $\theta_{0}$ is restricted to be les $s$ than $\pi / 2$ so that Donnell's shallow shell equations can be used.

$$
\begin{array}{r}
D \nabla^{2} w-\overline{\mathbb{N}}_{x} \frac{\partial^{2} W}{\partial x^{2}}-\frac{\vec{N}_{\theta}}{R^{2}} \frac{\partial^{2} w}{\partial \theta^{2}} \\
+\frac{1}{R} \frac{\partial^{2} F}{\partial x^{2}}+\rho h \frac{\partial^{2} w}{\partial x^{2}} \\
+p(x, \theta, t)=0 \\
\nabla^{4} F-\frac{E h}{R} \frac{\partial^{2} W}{\partial x^{2}}=0
\end{array}
$$

where $D$ is the bending rigidity of the shell, $\bar{N}_{X}$ and $\bar{N}_{\theta}$ are constants representing the components of membrane stress due to internal pressurization and $F(x, \theta, t)$ is the stress function defined so that

$$
\begin{aligned}
& \tilde{\mathrm{N}}_{\mathrm{x}}(\mathrm{x}, \theta, t)=\frac{1}{\mathrm{R}^{2}} \frac{\partial^{2} \mathrm{~F}}{\partial \theta^{2}} \\
& \widetilde{\mathrm{N}}_{\theta}(\mathrm{x}, \theta, t)=\frac{\partial^{2} \mathrm{~F}}{\partial \mathrm{x}^{2}}
\end{aligned}
$$

Note that $\widetilde{\mathbb{N}}_{x}$ and $\widetilde{\mathbb{N}}_{\theta}$ are the time dependent components of membrane stress due only to panel motion. The total membrane stresses are

$$
\begin{aligned}
& \mathrm{N}_{X}(x, \theta, t)=\bar{N}_{x}+\widetilde{N}_{x}(x, \theta, t) \\
& \mathbb{N}_{\theta}(x, \theta, t)=\bar{N}_{\theta}+\widetilde{N}_{\theta}(x, \theta, t)
\end{aligned}
$$


Boundary conditions to be applied at $x=0, x=I$ are

$$
v=w=\frac{\partial^{2} w}{\partial x^{2}}=\frac{\partial^{2} F}{\partial \theta^{2}}=0
$$

Boundary conditions at $\theta= \pm \frac{\theta_{0}}{2}$ are

$$
w=\frac{\partial^{2} w}{\partial \theta^{2}}=u=\frac{\partial^{2} F}{\partial x^{2}}=0
$$

The freely-supported boundary conditions have been chosen primarily because they are satisfied (term by term) by the series

$$
\left.\begin{array}{rl}
w(x, \theta, t)= & e^{i \omega t} \sum_{m=1}^{N} \theta_{m} \cos \frac{n \pi t}{\theta_{0}} \sin \frac{m \pi x}{\ell} \\
& 0 \leq x \leq L \\
& \frac{-\theta_{0}}{2} \leq \theta \leq \frac{\theta_{0}}{2}
\end{array}\right)
$$

These boundary conditions result in a plate which is dynamically "weaker" than on elastically "ostrained or a clamped plate. Experience has shown that an elastically restrained or clamped panel needs to be less thick to prevent flutter than the freely supported panels studied here.

At this point, the structural problem has been posed. We need to find the aerodynamic pressures $p(x, \theta, t)$ generated at the panel surface.

\section{Aerodynamic Details}

A strong assumption on the aerodynamic pressures will be made. The pressure on a panel deflection

$$
w(x, \theta, t)=\epsilon e^{i \omega t} \cos \frac{n \pi \theta}{\theta_{0}} \sin \frac{m \pi x}{L}
$$

will be assumed to be

$$
p(x, \theta, t)=\epsilon e^{i \omega t} \frac{\rho U^{2}}{\sqrt{M-1}} \frac{m \pi}{L} \cos \frac{n \pi \theta}{\theta_{0}} \cos \left(\frac{m \pi x}{L}+t\right)
$$

In other words, the rressure will have a magnitude equal to that given by Ackeret theory and a spatial. pressure distribution that can vary as desired.

The above approximation was made only after much thought about the physical situation and the theoretical solutions avalable. There are essentially three main features to be considered. First of all, this theory is quasi-static-it neglects the aerodynamic forces out of phase timewise with the displacement of the panel. Secondly, the spatial pressure distribution has been chosen is a simple trigonometric function which cannot account for the details of the panel geometry, e.g., the "leading edge effect." Thirdly, the amplitude of the forces has been chosen to correspond to that given by Ackeret theory (a short wave lencth theory) rather than by a slender boiy type of theory. Thess points will be discussed in turn.

The neglect of the out-of-phase forces is reasonable when one considers a system which flutter: in a "coupled rode" type of motion (rather than it single degrec-of-freedom) and if the frequericy involved is low. This is because multiple decree-offreedom instabilities are less sensitive to out-olphase forces than single degree-of-freedom instabilities. Also, when the flutter frequencies ar: low, the out-of-phase forces are small. Of courso, this would make the present analysis in error for axisymmetric flutter of cylinders, transonic ilutter and flutter of highly pressurized panels.

The simplification of the spatial pressure distribution simply must be done in order to get some results. In practice, the various geometries which should be covered include panels which are bounded by channels extending into the free stream and irclude panels forming hat sections. This precludes any exact analysis. Theoretical. sudies by Platzer, Beranek, and Saunders $(7)$ do indicate that for steady flow over an axisymmetric wavy cylinder at supersonic Mach numbers, the leading edge effect is usually small. Once one assumes a sinusoidal pressure waveform, as is done here, it then follows that the phase angle $\psi$ must lie between $0^{\circ}$ and $90^{\circ}$, on the basis of extensive numerica]. work on potential flow over wavy cylinders. (3)

Finally, the magnitude of the aerodynamic pressure expression was chosen to correspond to a short wave length theory because this represents an intermediate value. For certain wave patterns on a cylinder, a type of aerodynamic "resonance" (reinforcement of pressures along Mach lines) occurs and pressures develop which are larger in magnitude than indicated by Ackeret theory. (8) For long wove length ratios (very low aspect ratio wave patterns) the pressures drop below the values given by Ackeret theory. There are reasons to believe that neither extreme is reached in the case of a finite panel. Because of its finite wiath, the panel does not have enough repeated waves in the transverse direction to allow the "resonance" to occur, and hence high pressure magnitudes are not expected. On the other hand, existing solutions for low aspect ratio flat plates indicate that the instability involves eigenfunctions with short wave length content, and hence very long wave length solutions are not expected. (9)

The above reasoning led to the choice of the aerodynamic pressure expression in equation (11). The parameter $\psi$ is allowed to vary to account for the uncertainty in the pressure distribution. By 
letting $\psi$ vary from $0^{\circ}$ to $90^{\circ}$, one is essentially changing the pressure distribution from that of a two-dimensional supersonic theory to that of a slender body theory.

\section{Stability Details}

Galerkin's method is used to pose the problem in matrix form. The deflections of the shell - egment are

$$
w(x, \theta, t)=e^{1 \omega t} \cos \frac{n \pi \theta}{\theta_{0}} \sum_{m=1}^{N} a_{m} \sin \frac{m \pi x}{L}
$$

Note that this expression allows $n$ half waves in the circumferential direction of the panel. If $n$ takes a value higher than $l$, then the effective length-to-width ratio of the panel increases accordingly because there are stationary nodal lines down the length of the panel.

The expression for pressure, equation (II) is used in confunction with equations (1) and (2) to yield the set of linear algebraic equations of motion:

$$
\begin{gathered}
\sum_{m=1}^{N} a_{m}\left[\left\{\left[m^{2}+\left(\frac{L}{W e f f}\right)^{2}+\frac{12 z^{2} m^{4}}{\pi^{4}\left[m^{2}+\left(\frac{L}{W}\right)_{e f f}^{2}\right]^{2}}+\frac{N_{x} L^{2}}{\pi^{2} D} m^{2}\right.\right.\right. \\
\left.+\frac{N_{\theta^{I}}^{2}}{\pi^{2} D}\left(\frac{L}{W}\right)^{2}-\lambda-\frac{24 m \pi}{\pi^{4} H^{3}} \sin \psi\right\} \delta_{q m} \\
\left.+\frac{24}{\pi^{2} H^{3}} \eta_{q m} \cos \psi\right]=0 \\
(q=1,2, \ldots n)
\end{gathered}
$$

where:

$$
\begin{aligned}
H & =\left[\frac{\sqrt{M^{2}-1}}{\left(1-v^{2}\right)} \frac{E}{q}\right]^{I / 3} \frac{h}{I} \\
Z & =\frac{L}{R} \frac{L}{h} \sqrt{I-v^{2}} \\
L / w_{e f f} & =\frac{L n}{R \theta_{0}} \\
\lambda & =\frac{\rho_{s}^{h} \omega^{2} L^{4}}{\pi^{4} D} \\
\eta_{\mathrm{qm}} & =\left\{\begin{array}{cc}
0 & m+q \text { even } \\
\frac{4 q m}{q^{2}-m^{2}} & m+q \text { odd }
\end{array}\right\}
\end{aligned}
$$

Thus, a set of linear algebraic equations are obtained. The accurrence of a negative eigenvalue $\lambda$ signifies stat1c divergence of the panel and complex $\lambda$ signifies flutter.

\section{Results}

Stabllfty boundaries have been calculated for the aerodynamic loading discussed above. All results will be given for cases with zero membrane stresses $\bar{N}_{x}$ and $\bar{N}_{\theta}$.

The present study emphasizes the role played by the spatial pressure distribution. Because it is not clear how the value of $\psi$ affects the stabillty boundaries, some preliminary qualitative studies were carried out in Ref. 10. Let us consider one of the results of this earlier work.

Flrst of all, it should be remembered that under the present theory, there is no difference between the flutter of a shell segment and the standing wave flutter of a complete cylinder. The current theory allows no aerodynamic, elastic or inertial coupling of adjacent panels. Hence, a complete cylinder could flutter in a mode with adjacent panels oscillating independentiy.

The case recalied for 1llustration corresponds to the cylinder studied experimentally by olson. (2) The ratio of cylinder length to effective width $\mathrm{L} / \mathrm{W}$ eff was 9.2 and the cylinder curvature $(L / R)(L / h)\left(1-v^{2}\right)^{1 / 2}$ was 6,950 . When a cylinder of this geometry is studied under the present theory, the effect of a variation in $\forall$ is found as is shown in Figure 5 . The dynamic pressure required to cause flutter or divergence is plotted. It is seen that for $\psi$ between $0^{\circ}$ and $60^{\circ}$, only flutter is predicted. Furthermore, the flutter boundary does not vary greatly with $\psi$ in this range. For $\psi$ between $60^{\circ}$ and $90^{\circ}$, one observes flutter at moderate dynamic pressures and divergence at high dymamic pressures.

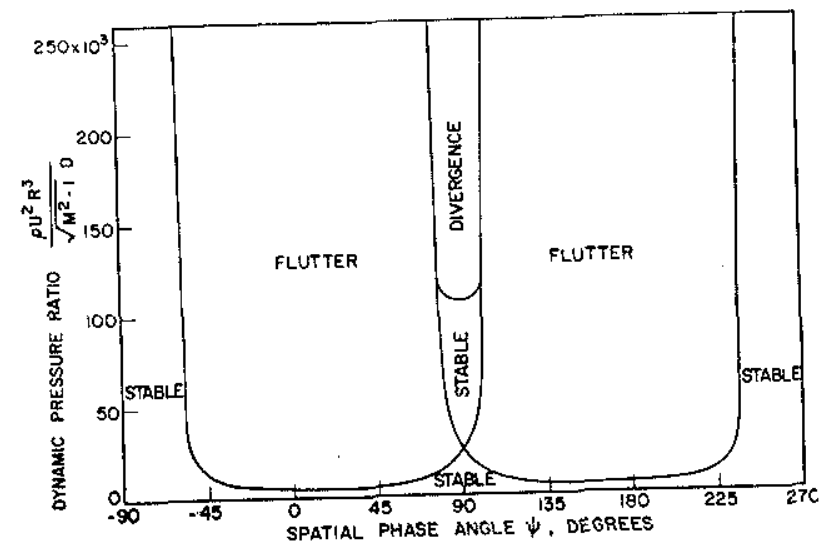

Figure 5. Stablilty boundaries for a cylinder. From Ref. 10.

In the results that foliow, a decision had to be made as to how to present the data. It was 
decided to solve for flutter boundaries taking $\psi=0^{\circ}$ and $\psi=90^{\circ}$ as the two cases of greatest interest. This is somewhat an arbitrary choice, since the critical thickness requirement lies near $30^{\circ}$ in the example just studied. The case at $\psi=90^{\circ}$ is unusual in the fact that no flutter is predicted for $\psi$ preclsely equal to $90^{\circ}$. (It can be seen that the flutter natrix is Hermitian and cannat hare complex eigenvalues for this case.) The occurrence of flutter at nearby values of $\psi$ is ominous and hence the "flutter boundary" at $\psi=90^{\circ}$ is taken as the point on the figure where the two flutter boundarles intersect (the limiting value as $\psi$ goes to $90^{\circ}$ ).

Stability boundaries for shell segments are given in Figures 6-9. The results are presented using an effective length-to-width ratio $\mathrm{L} / \mathrm{W}_{\text {eff }}$ the curvature parameter $\mathrm{z}=(\mathrm{L} / \mathrm{R})(\mathrm{L} / \mathrm{h})\left(1-v^{2}\right) 1 / 2$, and a thicikness parameter $H=[\sqrt{\mathrm{M}-1} \mathrm{E} /$ $(1-v) q]^{1 / 3} h / L$. The plots of $H$ versus $L /$ werf are given as a generallzation of the work of Kordes, Tuovila, and Guy and the curvature parameter $Z$ corresponds to a parameter used in the study of cylinder buckling. (11,12) There are two subtle points about these parameters. First, the effective length-to-width ratio is not unique for a given panel. A panel with given geometric length and with may flutter in a mode with $n>1$. Hence, a penel may have an effective length-towidth ratio which is any multiple of its geometric length-to-width ratio. Second, both parameters H. and $Z$ contain the thickness of the panel. Depending on the way the design curves are used, this can cause a small inconventence (requiring a simple iteration to get, a useful result when one wishes to solve for a required thickness). As presented, the design curves are most straightforwardly used when one knows the physical properties of a panel and wishes to check 1ts stab1lity.

It was found that for $L / W$ eff large and for small curvature, many modes were needed for convergence. Gaspars and Redd studied carefully the number of modes required for convergence on finite aspect ratio flat plates when Ackeret theory is used. (9) They found that as many as 50 modes were needed for flat plates with aspect ratios of 10 . or more. When curvature is present, the results do not seem to be as 'sensitive to convergence problems. It was felt that 30 modes were sufficlently accurate for this study.

The solution for $\psi=0^{\circ}$ (Ackeret theory) is given in Figure 6 . It is easily seen that curvature helps to stiffen the panel and reduce the thickness requirement. An interesting effect is obtained in the regions where $H$ increases with increasing $\mathrm{L} / \mathrm{Weff}$ (positive slope). This is the case where a panel of given physical length and width will flutter in a mode with $n>1$, giving a higher critical value of $\mathrm{L} / \mathrm{W}_{\text {eff. }}$. As an example, a panel of length 10 inches and width 2 inches has a physical length-to-wiath ratio of 5 . If $Z=8000$ for this panel, then it might appear that a thickness ratio of 0.034 would be sufficient to prevent flutter. One must consider, however, all multiples of 5 as possible effective length-to-width ratios. one then sees that $\mathrm{L} / \mathrm{W}_{\text {eff }}=20$ yields a thickness requirement of $\mathrm{H}=0.055$. This particular panel flutters with $\mathrm{n}=4$, i.e., it has three interior nodal lines extending down its length.

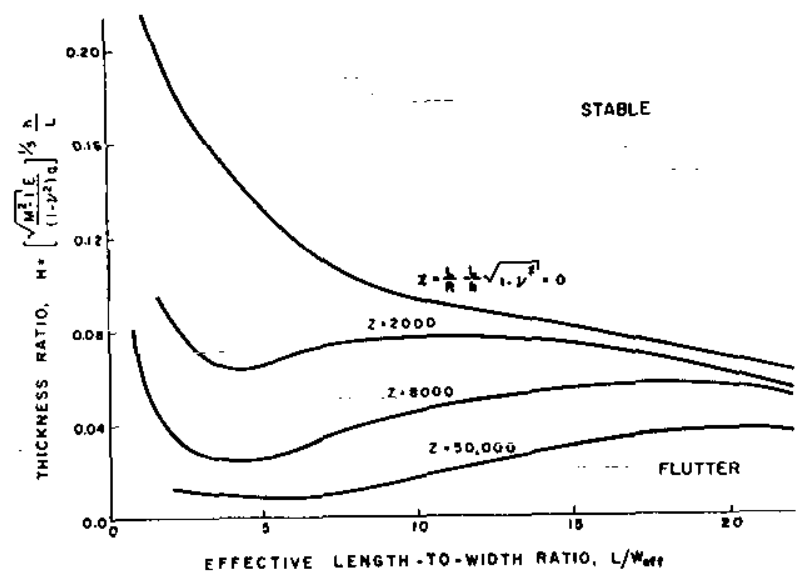

Figure 6. Stablilty boundaries. Ackeret aerodynamic theory.

Results for $\psi=90^{\circ}$ are given in Figure 7 . These results are similar to the $\psi=0^{\circ}$ case except that the instabilities in the lower left corner are due to static divergence. Again, one must observe the cases where $\mathrm{H}$ increases with $\mathrm{L} / \mathrm{W}_{\text {eff }}$ and one must choose the multiple of the geometric lengthto-width ratio which gives the largest value of $\mathrm{H}$.

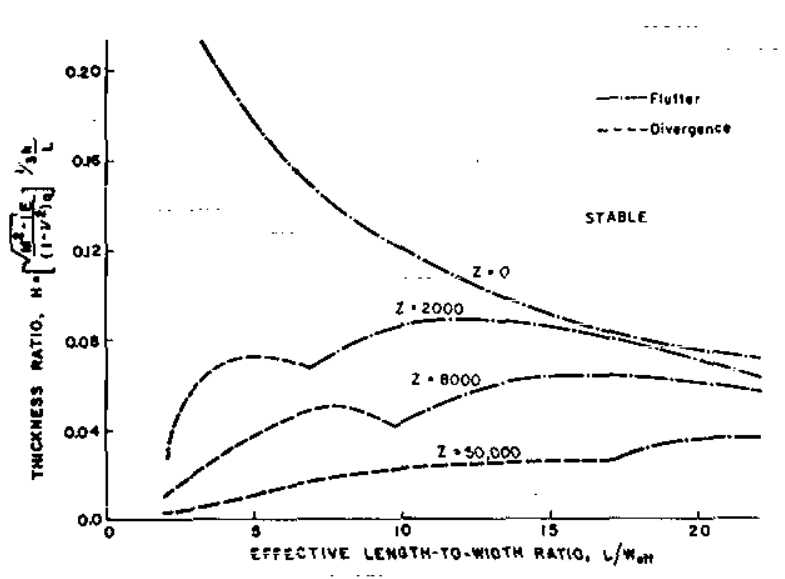

Figure 7. Stability boundaries. "'slender body" aerodynamic theory. 
The most useful results, from a design standpoint, are given in Figure 8. This figure combines the results for $\psi=0^{\circ}$ and $\psi=90^{\circ}$. Note that the difference between the two bounding curves is not great, part1cularly in certain intermediate regions of $L / W_{\text {eff }}{ }^{\circ}$ This may be an indication why Ackeret theory gives relatively good results for the cylinder experiments discussed in Refs. 1 and 2 (see next figure). As experiments are carried out, corrections can be applied to design figures such as this and some confidence in their accuracy can be established.

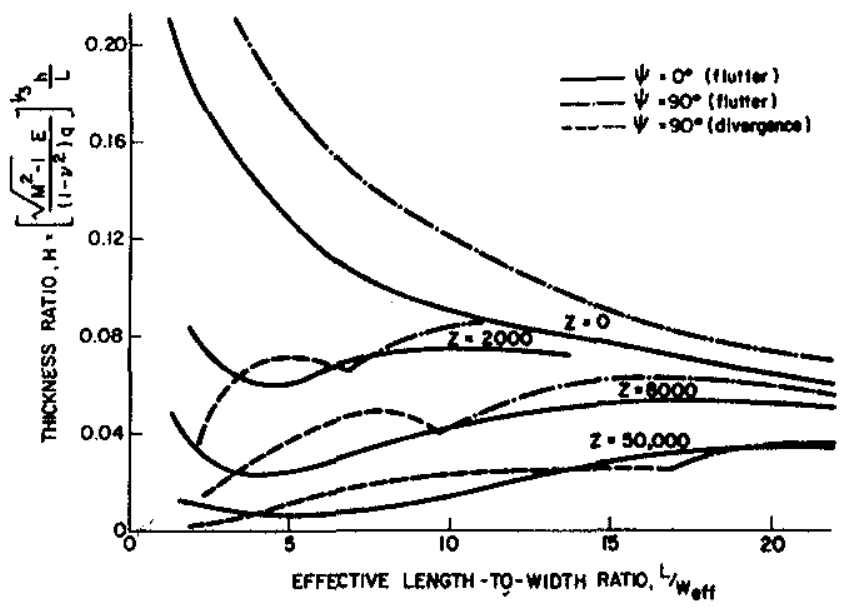

Figure 8. Upper and lower bounds for thickness requirement. Freely supported ledges.

Other theories and experiments are shown in Figure 9. Several of the points correspond to work for full cylindrical shells. Structurally, the major difference is that a complete, unstiffened cylinder can flutter in modes with waves travelling in the circumferential direction whereas the segment cannot. of particular interest in Figure 8 are the experimental points found for full cylinders by Steamen, Lock, and Fung, and by 01 son and Fung. $(1,2)$ It is now believed that these cylinders did flutter in clrcumferentially travelling waves. (13) Theoretical nonlinear flutter boundaries found by Evensen and olson give a larger thickness requirement than the current linear study. This may explain why the experimental values occurred at slightly higher values of thickness ratio than predicted by the present theory.

The experiments of Tuovila and Hess were corried out for a shell segment clamped all around. (14) The tests were done at Mach 2.3, wh1ch unfortunately brings transonic effects into the comparison. In transonic flow, the unsteady aerodynamic terms are of importance and these terms are missing in the present theory.

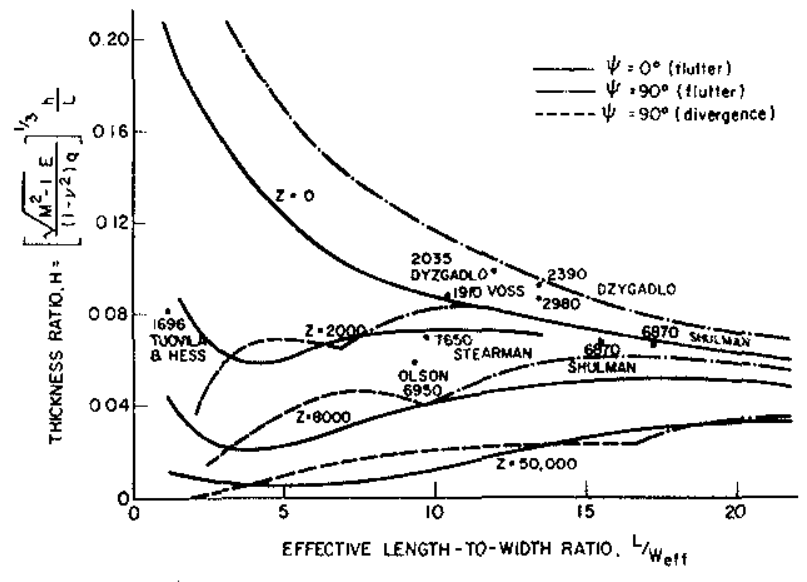

Figure 9. Comparison with other theories and experiments.

The theorles of Voss and Shulman both were cone for a complete cylinder with the use of Ackeret theory. $(15,16)$ These should (and do) correspond"with the present calculations and serve as a check. Their results yield thickness ratios H alightly higher than the present results. This reflects the fact that fewer modes were used. (For low curvatures and large length-to-width ratios, the thickness requirement converges from above.)

There are no unclassifled experiments known to the authors which furnish the proper comperison with the theory. Such tests would be useful.

\section{Conclusions}

A simplified study of the aeroelastic instebillty of curved panels was carried out. The assumptions made were based on theoretical solutions for flow over stationary wavy walls. A parameter included to account for the uncertai tainty in the aerodymaric pressure distribution on the panel. Upper and lower estimates for the flutter boundaries were presented. It was found possible to summarize most of the information for curved, unstressed panels in one design figure. Comparisons with other theories and experiments make the present analysis look reasonable, although detafled comparisons are impossible because of differences in the problems studied.

The design curves are relatively easy to use and should be suttable for rough estimates of flutter boundaries. They should be particularly helpful for wind tunnel testing, where one is interested in both upper and lower estimates for the flutter boundary. In using the design curves, one showld remember to check the stability not only at the given geometric length-to-width ratio, but also at higher multiples of this ratio. 


\section{References}

(1) Stearman, R.O., Lock, M. H., and Fung, Y. C., "Ames Tests on the Flutter of Cylindrical. Shells," GALCIT Structural. Dynamics Report SM 6237, December 1962.

(2) OIson, M. D. and Fung, Y. C., "On Comparing Theory and Experiment for the Supersonic Flutter of Circular Cylindrical Shells," AIAA 5th Aerospace Sciences Meeting, AIAA Paper No. 67-77, New York, January 1.967.

(3) Dzygadlo, z, "The Problem of Aeroelasticity of a Cylindrical Panel and a Plate Strip Taking into Consideration the Transversal Coupling," Proceedings of Vibration Problems, Warsaw, 2, 5 (1964).

(4) Dowel1, E. H. and Wianall, S. E., "General1zed Aerodynamic Forces on an Oscillating Cylindricol She11," Quart. Appl. Mech., XXIV, 1, April. 1966.

(5) Randall, D. G., "Supersonic Flow Past Quasi-Cylindrical Bodies of Almost Circular Cross Section," British R of M No 3067, November, 1.955.

(6) McElman, J. A., "Flutter of Curved and Flat Sandwich Panels Subjected to Supersonic Flow," NASA TN D-2192, Langley Research Center, April 1964.

(7) Platzer, M., Bermnek, R., and Saunders, L., "On Some Aerodynamic Aspects of the Panel. Flutter Problem," TNX 53389, Marshall Space Flight Center, Huntsville, Ala., October, 1.965.

(8) Anderson, William J., "Oscillatory Pressures in a Simplified Boundary Layer with Application to Cylinder Flutter," AIAA Journal, Vol. 4, No. 5, May li966, pp. 865-872.

(9) Gaspars, P. A., and Redd, B., "A Theoret1cal Analysis of the Flutter of Orthotropic Panels," NASA TN D-355.1, Ames Research Center, August 1966.

(10) Anderson, William J., "Aeroelastic stability of Plates and Cylinders." The University of Michigan Report 08079-2-F, Part A. Also: Proceedings of the Fifth Southeastern Conference on Theoretical and Applied Mechanics, February 29, 1968, Pergamon Press, N.Y.

(11) Kordes, E. E., Tuovila, W. J., and Guy, L. D., "Flutter Research on Skin panels," NASA TN D-451, Langley Research Center, Sept. 1960.

(12) Batdorf, S. B., "A Simplified Method of Elistic-Stability Analysis for Thin Cylindrical Shells," NACA Report 874, Langley Research Center, 1947.

(13) Evensen, D. A., and Olson, M. D., "Circumferentially Traveling Wave Flutter of a Circular Cylinarical. Shell, "To be presented: AIAA 6th Aerospace Sciences Meeting, New York, January, 1.968.

(14) Tuovila, W. J., and Hess, R. W., "Experimental Investigation of Flutter of Buckled, Curved Panels," NASA Memo 5-18-59L, Lang]ey Research Center, June 1.959.

(15) Voss, H. M., "The Eff'ect of an External. Supersonic Flow on the Vibration Characteristics of Thin Cylindrical Shells," J. Aerospace Sci., Vo.1. 28, No. 12, December 1961.

(16) Shulman, Y., "Vibration and Flutter of Cylindrical and Conical. Shells," OSR Technical Report No. 59-776 Massachusetts Institute of Technology, June 1959.

(17) Dzygedlo, Z., "Self Excited Vibration of" a Cylindrical shell. of Finite Length in Supersonic Flow, "Proceedings of Vibration Problems, Warsaw, Vol. 3, No. 1., 1.962 . 